\title{
Post-ACL Rupture Integrated Rehabilitative Pathway: Factors Influencing Economic Resources Absorption
}

\author{
Lorella Cannavacciuolo ${ }^{1}$, Emanuela Foglia $^{2}$, Lucrezia Ferrario ${ }^{2 *}$, Daniele Bellavia ${ }^{2}$, Davide \\ Crisci $^{2}$, Federica Asperti ${ }^{2}$ and Cristina Ponsiglione ${ }^{1}$ \\ ${ }^{1}$ Department of Industrial Engineering, University of Naples Federico II, Italy \\ ${ }^{2}$ Centre for Health Economics, Social and Health Care Management, LIUC-Università Cattaneo, Italy
}

*Corresponding author: Lucrezia Ferrario, Centre for Health Economics, Social and Health Care Management, LIUC-Università

Cattaneo, Castellanza, Corso Matteotti 22, 21053, Castellanza (VA) - Italy

\section{ARTICLE INFO \\ Received: 幽 January 10, 2021 \\ Published: 彗 January 20, 2022 \\ Citation: Lorella Cannavacciuolo, Ema- nuela Foglia, Lucrezia Ferrario, Daniele Bellavia, Davide Crisci, et al., Post-ACL Rupture Integrated Rehabilitative Path- way: Factors Influencing Economic Re- sources Absorption. Biomed J Sci \& Tech Res 41(2)-2022. BJSTR. MS.ID.006564.}

Keywords: Real-world data; healthcare sector; rehabilitative pathway; regression analysis; Qualitative Comparative Analysis

\section{ABSTRACT}

Purpose: To investigate the drivers that might influence the patient's integrated rehabilitative pathway, after the rupture of the anterior cruciate ligament (ACL), in terms of economic resources absorption.

Materials and Methods: An observational study, conducted in two private rehabilitation hospitals located in Italy, was conducted to obtain real-world data concerning the integrated rehabilitative pathway of 118 patients. Patients' data were analysed adopting three methodological approaches.

i) Relationships between variables, looking for correlations among them.

ii) A hierarchical sequential linear regression model, to define the predictors of the dependent variable.

iii) A Qualitative Comparative Analysis to complement previous statistical approaches, with a comparative-configurational one.

Results: Results reported that older age $(\beta=0.145, \mathrm{p}=0.049)$, higher sportiness level $(\beta=0.169, p=0.022)$, as well as the length of the integrated rehabilitative pathway $(\beta=0.550, p$-value $=0.000)$ and the performance of threshold tests $(\beta=0.389, p$-value $=0.000$ ) are antecedents of higher management costs (Adjusted $R^{2}=0.703$ and $\mathrm{F}=24.482$ ). Summarizing QCA results, age, and the level of sportiness of patients, in combination with the compliance, play an important role in the emergence of the selected and analyzed management costs.

Conclusions: Findings could be useful for the optimization of integrated rehabilitative pathway, thus becoming more efficient and effective, guaranteeing a process personalization, consistent with efficiency-driven and cost-containment strategies.

\section{Introduction}

In the healthcare setting, randomized controlled trials (RCTs) usually represent the main approach to create new knowledge [1]. Nowadays the use of real-world data (RWD) is becoming more and more relevant, even from a strategic point of view, to optimize and determine the best patients' pathway. Using RWD gives the opportunity to generate new knowledge assets, mixing points of view from different actors (i.e. hospitals or patients) together with outcome and efficiency data, [2,3] thus supporting the delivery of 
healthcare services improvement and the outcomes' optimization [4]. In this growing scenario, where RWD assume more and more importance all around the world, there is the opportunity to gather different dimensions and information, to create larger and integrated databases. Subsequently, they can be elaborated and merged at different levels, and could become available for both scholars, and policy makers research, thus achieving improvements in the quality, safety, and value of healthcare services delivery.

RWD could thus represent a useful support tool to assess the consequences of disease management interventions, to design an integrated clinical pathway based on knowledge derived from real world data and facts, implementing, and improving, the healthcare services that are satisfactory, in terms of effectiveness and efficiency [5]. The use of RWD could also provide a robust support for making customized decisions, concerning the treatment of patients, and for developing strategies for the delivery of care that respect the principles of fairness and coverage, without overshadowing the dimensions of quality and services efficiency/productivity. One of the most important knowledge assets that should leverage on RWD, are the costs related to specific rehabilitative programs or integrated clinical and rehabilitation pathways, [6] that could rely on real-life and specific country-oriented evidence, always following international and national guidelines suggestions and indications, and for which poor evidence is still available.

According to the above, integrated clinical and rehabilitative pathway could be the perfect example to define the significance to observe real-world healthcare data, beyond clinical trial evidence, thus providing stakeholders with valuable information about the safety and effectiveness of rehabilitative programs in a large and heterogeneous populations. Rehabilitation is an essential part of universal health coverage along with promotion of good health, prevention of disease, treatment, and palliative care, thus being defined as "disability in individuals with health conditions in interaction with their environment" [7]. The above consideration is strengthened because literature evidence focused their attention, on the inpatient's integrated clinical pathway, without considering the outpatients' activities and programs, after discharge. Moving on from these premises, the present paper aims at investigating the knowledge assets that might influence the creation and the design of a proper patient integrated rehabilitative pathway, after the Anterior Cruciate Ligament (ACL) rupture, assuming the hospital's perspective. Coherently to the above, the study addressed the following two research questions.

1. Which are the main factors influencing the efficiency of the integrated rehabilitative pathway, in terms of costs' optimization?
2. What configurations of such factors are sufficient to generate the emergence of a certain level of efficiency, in the absorption of resources?

\section{Theoretical Backgrounds}

The deep investigation about the above research questions requires the analysis of different independent variables, and their relations with the optimization of the entire integrated clinical and rehabilitative pathway. Based on an extensive literature review, a set of original hypotheses was developed, to produce a specific framework, declaring the main characteristics of a rehabilitative pathway that affect the absorption of economic resources: patient's age, patient's compliance, duration of the rehabilitation pathway, level of sportiness, number of physiotherapy sessions performed, number of muscle strength and threshold tests performed were the factors investigated [8-12].

\section{Age}

De Valk stated that age is a key factor for the success of the integrated rehabilitative pathway: younger patients are more likely to achieve a better clinical outcome, with a positive impact on satisfaction [8]. Since, in other contexts, younger patients are usually associated with a lower absorption of economic resources, [13] it is possible to assume that also in this case age could be a factor able to reduce the overall costs of the clinical rehabilitation pathway. Based on the above considerations, the following hypothesis was displayed.

- HP 1: Younger age has a positive impact on rehabilitative pathway management costs.

\section{Level of Sportiness}

As reported in the study of De Valk et al., a high level of sportiness enables a fast and successful integrated rehabilitative pathway [8]. Patients who are used to practice sports at high levels (e.g. professional athletes), are more likely to fully recover knee functions, achieving levels of mobility and resistance to pre-injury efforts The level of sportiness, therefore, has a strong impact on the outcome of rehabilitation, and consequently also on the resources' absorption as well as on the rehabilitation pathway efficiency.

The following hypothesis was formulated.

- HP 2: High level of sportiness, positively impacts on rehabilitative pathway management costs.

\section{Compliance}

Patient compliance is a fundamental component of rehabilitation activity of which the main goal is improved recovery outcome. Without compliance to the treatment regimen, the expected 
outcomes cannot be achieved. According to this consideration, one of the most frequent obstacles to the healthcare professionals work, is low level of compliance and adherence in all integrated clinical pathways, but particularly in physical rehabilitation.

In fact, many patients do not follow the instructions of the clinicians and physiotherapists, who plan the timing and exercises of rehabilitation sessions, and often do not respect them or do them partially. Literature estimated that sport injury rehabilitation adherence rates could be as low as $40 \%$. [14-16]. On the contrary, being adherent to the integrated rehabilitative pathway plays a key-role in the achievement of the expected results, with a positive impact in the overall economic resources absorption [17].

Thus, the following hypothesis was proposed.

- $\quad$ HP 3: Compliance has a positive impact on rehabilitative pathway management costs.

\section{Length of the Rehabilitative Clinical Pathway}

The duration of the rehabilitative pathway presents a direct impact on the overall clinical results, and it is closely related to adherence [12]. In general terms, the presence of adequate clinical pathway would enhance the overall patients' journey optimization. [18] Moving on from the above consideration, the more the patients are satisfied and adhere to the proper appointments, fewer follow-up activities would be required, with a positive impact on the possibility to free up economic resources [19]. The following hypothesis was defined.

- HP 4: The length of rehabilitation negatively impacts on rehabilitative pathway management costs.

\section{Number of Physiotherapy Sessions Performed and Number of Muscle Strength Tests}

Two other variables related to the overall duration of the integrated rehabilitative pathway, impacting on the rehabilitation management costs, are the number of sessions and the number of muscle strength tests performed. Risberg and colleagues (2004) revealed a correlation between prolonged rehabilitation and improvement in patient's physical conditions, leading to increased costs to support intensive rehabilitation programs [20].

According to this, the following hypotheses were displayed.

- HP 5: The number of physiotherapy sessions negatively impact on rehabilitative pathway management costs.

- HP 6: The number of muscle strength tests negatively impact on rehabilitative pathway management costs.

A synthesis of the research frameworks developed, is proposed in Figure 1.

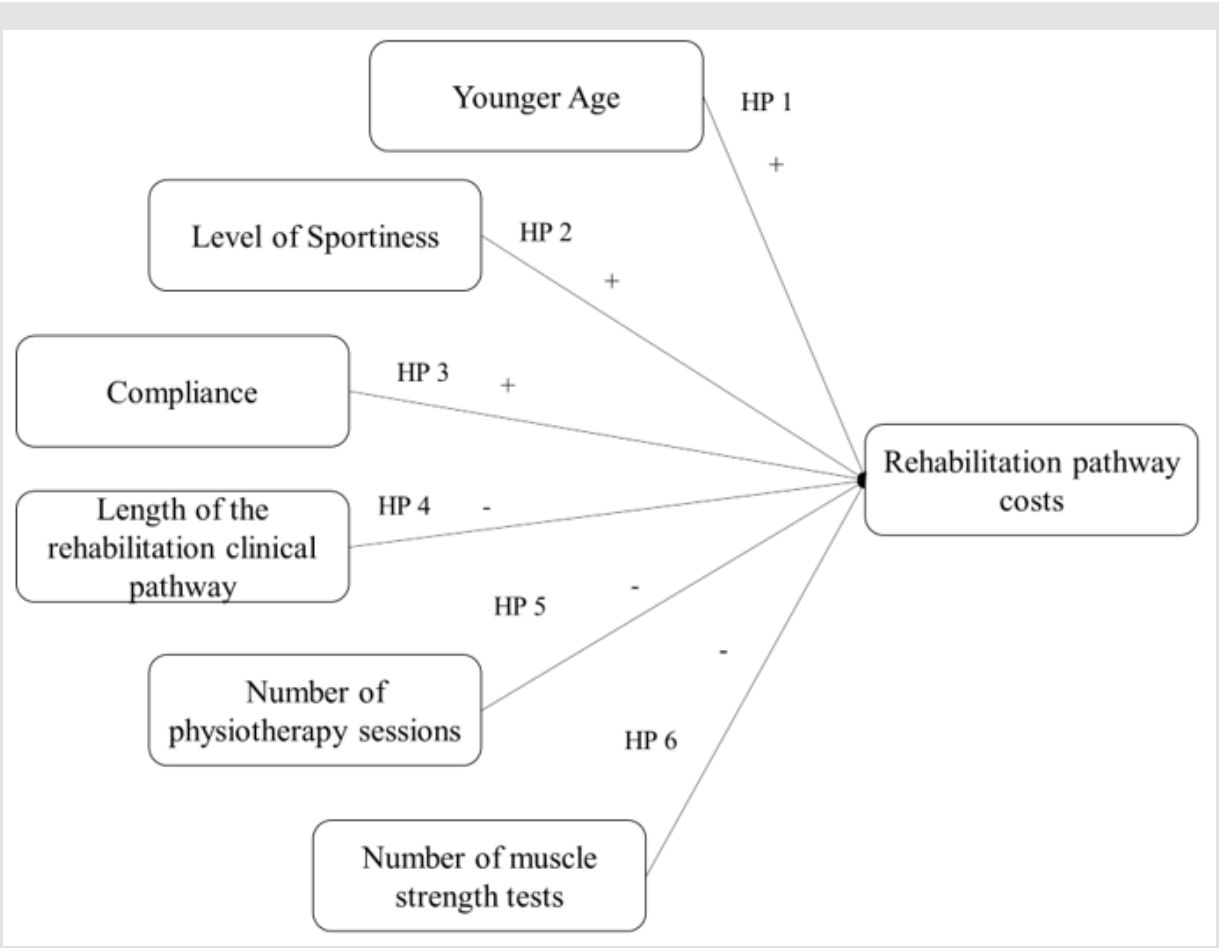

Figure 1: Conceptual framework for rehabilitative pathway costs. 


\section{Methods}

An observational study, designed in accordance with the STROBE guidelines, [21] was conducted in two private rehabilitation hospitals in Italy, involving a total of 118 patients, to retrieve RWD, in relation to the integrated rehabilitative pathway. The informed consent forms were signed during the taken in charge of the patients, and they were informed concerning the use of data with an anonymous and aggregated methodological approach. In particular, the study involved economic data referring to all the adult patients (age $\geq 18$ years old) requiring an integrated rehabilitative pathway after ACL rupture, in the year 2018, after having signed the informed consent form for dissemination activities.

\section{Definitions of the Measures}

As previously mentioned, the study was structured considering just one dependent variable, the rehabilitative pathway costs, and six independent variables, described below.

\section{Dependent Variable}

Costs: For the estimation of the integrated rehabilitative pathway average costs, the following variables were considered: the number of physiotherapy sessions and the number of procedures / tests performed during the entire programme. Economic data were evaluated, in accordance with outpatient visits and tests Regional Reimbursement Tariffs, valid for the years 2021/2022.

\section{Independent Variables}

Age: The patient's age when rehabilitation program begins.

Level of Sportiness: It is the declared level of sportiness when the integrated rehabilitative pathway begins, defined by clinician and physiotherapist taken in charge the patient, in terms of number of training sessions performed within a week. In particular, the level of sportiness was classified as:

1. None (no training sessions performed).

2. Practitioner (at least 1 or 2 training sessions a week).

3. Amateur (at least 3 or 4 training sessions a week).

4. Athlete (at least 4 or 5 training sessions a week, with participation at sports competitions).

Compliance: The adherence/compliance to the integrated rehabilitative pathway was esteemed by attendance and participation in scheduled sessions. Patients were classified as compliant patients and not-compliant patients, depending on these two cases:

1. Patients completed the pathway prescribed at the beginning, even considering any modifications in the integrated rehabilitative pathway, and
2. Patients abandoned the rehabilitative pathway earlier.

Length of the Rehabilitation Clinical Pathway: This variable indicates the duration of the pathway, expressed as number of days occurred between the beginning and the end of the integrated program.

Number of Physiotherapy Sessions Performed and Number of Muscle Strength: This variable indicates the number of tests and sessions performed by each patient, during the whole rehabilitation pathway.

\section{Statistical Analyses}

The above variables were first analysed considering descriptive statistics, frequencies, and distributions. To ensure the relevance of the assumptions of normality, linearity and homoscedasticity, preliminary analyses were carried out. Three methodological approaches were used to answer the research questions underlying the study.

1. An analysis was carried out on the relationships among variables, verifying the existence of correlations among them. In particular, the correlation coefficient "person-product-moment" was evaluated, to verify the existence of small (from 0.10 to 0.29 ), medium (from 0.3 to 0.49 ), or large (from 0.5 to 1 ) correlations [22]. The exact value of -1 or +1 indicated a perfect correlation among variables.

2. In order to test the proposed hypotheses, a hierarchical sequential linear regression model was implemented (with enter methodology), that defines the predictors of the dependent variable (level of economic resources' absorption). This approach was used to identify the impacts of the independent variables. One parameter of attention was the Adjusted R2, useful to control the explanatory power of each model. Thanks to this approach it is possible to test the hypotheses, through incremental models, to establish the specific impact of each input variable on the dependent variable. The "exclude case pairwise" option has been implemented, as it represents the preferred methodology to be applied for a small sample size, avoiding data exclusion. All the statistical analyses, referring to the coefficients test, and the development of the hierarchical sequential linear regression model were performed using the Statistical Package for Social Science (IBM SPSS Statistics Viewer - Version 22).

3. A qualitative-configurative analysis - QCA - was carried out to integrate the statistical approaches previously described $[23,24]$. The QCA is a comparative case-oriented methodology, useful to find, through an in-depth comparison of real-world cases, consistent configurations of causal conditions (the independent variables in our study), sufficient to determine the emergence of a specific outcome [25]. In the QCA, a symmetric relationship is 
disarticulated into two asymmetric analyses formalized by set and sub-set relationships [21]: one, related to the necessity of the conditions, with respect to the dependent variable, and the other on the sufficiency. This approach allows researchers to deal with the complexity of real phenomena. The QCA assumes the nonlinearity of phenomena under investigation and is based on the principle of causal complexity. This means that, in most cases, it does not make sense to isolate the effect (positive or negative), of a single independent variable on the outcome, but configurations of variables are identified, being related to the dependent variable. Moreover, several different configurations can be recognized as "causal recipes" of the same dependent variable [23]

In accordance with Vis, [26] which discussed the advantages of using the QCA technique to complement regression analyses for moderately large samples (between 50 and 100), the 118 empirical cases collected in this research were studied through the QCA to better understand the relationships between combinations of independent variables (the effects of complex interactions between causal conditions) and dependent variables, i.e. the results presented above. In QCA approach, the variables can be considered crisp or fuzzy. The crisp set variant (csQCA) is the version in which the variables of the study are dichotomous, and the empirical analysed cases are classified as alternatively "fully in" or "fully out" in the sets representing causal conditions and the outcomes. The fuzzy-set variant (fsQCA) is characterized by the fact that the empirical cases are classified in terms of membership degrees in the fuzzy sets of causal conditions, and of the outcome. In this analysis, we adopted the crisp set QCA.

\section{Results}

\section{The Sample Under Assessment}

The sample was composed of 118 patients, requiring a specific integrated rehabilitative pathway after ACL rupture. It should be noted here that all patients required an outpatient integrated rehabilitative pathway, starting the activities program immediately after surgery. The sample consisted mainly of males (66\%) and is 34 years old on average. As far as the level of sportiness is concerned, only $3 \%$ of the patients in the sample had previously practiced sport at competitive level, while $64 \%$ of the patients belonged to the "practitioner" category. In general, the average absorption of economic resources associated with the rehabilitation of the patients in the sample was equal to $€ 3,334.07 \pm 269.28$. Compliant patients are associated with a higher absorption of financial resources than non-compliant patients ( $€ 3,634.47$ vs $€ 2,157.50$ p-value $=0.026$ ).

\section{Hypotheses Testing}

Table 1 shows that age ( $\mathrm{p}$-value $=0.047)$, compliance $(\mathrm{p}$-value $=0.026)$, length of the rehabilitative pathway ( $p$-value $=0.000)$ and number of threshold tests ( $\mathrm{p}$-value $=0.000$ ) significantly influenced the integrated rehabilitative pathway costs. The great relationship between total costs and the number of physiotherapy sessions $(\beta=$ 0.995 , $p$-value $=0.000$ ) depicts that between the two variables there is the collinearity phenomenon, thus being two aspects explaining the same concept. This is the reason why the variable "number of physiotherapy sessions" was not included in the regression model. The independent variables (age, compliance, and the length of the rehabilitative pathway) have a significant $\mathrm{p}$-value $<0.05$. Moreover, the same relationships emerged among the length of the integrated rehabilitative pathway, the number of threshold tests and the number of physiotherapy sessions ( $p$-value $=0.000$ ). After testing the correlation among all variables, a regression analysis was conducted to test hypotheses (Table 2$)$. Older age $(\beta=0.145$, $p=0.049)$, higher sportiness level $(\beta=0.169, p=0.022)$, as well as the length of the rehabilitative pathway $(\beta=0.550, p$-value $=0.000)$, and threshold tests $(\beta=0.389$, $p$-value $=0.000)$ are antecedents of higher rehabilitative pathway management costs (Adjusted $\mathrm{R}^{2}=0.703$ and $\mathrm{F}=24.482$ ).

Table 1: Relationships between variables.

\begin{tabular}{|c|c|c|c|c|c|c|c|}
\hline & 1 & 4 & 5 & 6 & 7 & 8 & 9 \\
\hline $\begin{array}{l}\text { Integrated rehabilitative pathway } \\
\text { management costs (1) }\end{array}$ & 1 & & & & & & \\
\hline Age (2) & $0.260^{*}$ & 1 & & & & & \\
\hline Compliance (3) & $0.290^{*}$ & $0.565^{* *}$ & 1 & & & & \\
\hline Level of sportiness (4) & 0.244 & -0.195 & 0.015 & 1 & & & \\
\hline $\begin{array}{l}\text { Length of the integrated rehabilitative } \\
\text { pathway (5) }\end{array}$ & $0.735^{* *}$ & $-0.265^{*}$ & 0.252 & 0.082 & 1 & & \\
\hline Number of threshold tests (6) & $0.637^{* *}$ & 0.043 & $0.373^{* *}$ & 0.062 & $0.403^{* *}$ & 1 & \\
\hline Number of physiotherapy sessions (7) & $0.995^{* *}$ & $0.477^{* *}$ & $0.270^{*}$ & $0.259^{*}$ & $0.738^{* *}$ & $0.559^{* *}$ & 1 \\
\hline
\end{tabular}


Table 2: Regression model for integrated rehabilitative pathway management costs.

\begin{tabular}{|c|c|c|c|c|c|}
\hline \multirow{2}{*}{} & \multicolumn{3}{|c|}{ Rehabilitative Pathway Management Cost } \\
\cline { 2 - 6 } & Mod 1 & Mod 2 & Mod 3 & Mod 4 & Mod 5 \\
\hline Age & $0.260^{*}$ & $0.239^{\wedge}$ & $0.230^{\wedge}$ & $0.172^{*}$ & $0.145^{*}$ \\
\hline Compliance & & $-0.271^{*}$ & $-0.268^{*}$ & -0.104 & 0.008 \\
\hline Level of sportiness & & & $0.231^{\wedge}$ & $0.180^{*}$ & $0.169^{*}$ \\
\hline Length of the integrated rehabilitative pathway & & & $0.675^{* *}$ & $0.550^{* *}$ \\
\hline Number of threshold tests & & & $0.389^{* *}$ \\
\hline R2 & 0.068 & 0.141 & 0.194 & 0.615 & 0.729 \\
\hline Adj. R2 & 0.051 & 0.11 & 0.15 & 0.587 & 0.703 \\
\hline F value & $4.134^{*}$ & $4.587^{*}$ & $4.412^{*}$ & $21.576^{* *}$ & $28.482^{* *}$ \\
\hline$\Delta \mathrm{R} 2$ & 0.068 & 0.073 & 0.053 & 0.421 & 0.114 \\
\hline F $(\Delta \mathrm{R} 2)$ & $4.134^{*}$ & $4.766^{*}$ & $3.632^{\wedge}$ & $59.089^{* *}$ & $22.208^{* *}$ \\
\hline
\end{tabular}

\section{Configuration of Factors Through QCA}

The QCA analysis requires a preliminary step for calibration of variables transforming them in crisp ones (Table 3). After performing the calibration process, consistency, and coverage of a set of configuration variables were evaluated. Consistency is the extent to which the results are in line with statements of need or sufficiency. Coverage provides information about the empirical importance of necessary and sufficient conditions. Each configuration has a raw coverage value that "measures the relative importance of several combinations of causally relevant conditions" [23]: the proportion that a configuration covers the outcome. It is assessed by the sum of consistent scores of the configuration divided by the sum of outcome scores [27]. In the QCA analysis, the crisp value 1 is reported in uppercase whereas the crisp value 0 is reported in lowercase. According to the regression analysis, the variables that most influence the management costs are the compliance and the length of the integrated rehabilitative pathway (Table 4). In particular, the most representative recipes show that level of sportiness associated with young age affects the management, as well as the length of rehabilitation, also for the patients with a lower level of sportiness.

Table 3: Calibration of the variables.

\begin{tabular}{|c|c|c|}
\hline Variable & Value in Regression Model & Calibration \\
\hline \multirow{4}{*}{ Age } & a) 50-60 years & $0:<30$ years \\
\hline & b) 30-49 years & 1: 49-60 years \\
\hline & c) 20-29 years & \\
\hline & d) $<20$ years & \\
\hline \multirow{4}{*}{ Level of sportiness } & a) none & 0: none and practitioner \\
\hline & b) practitioner & 1: amateur and athlete \\
\hline & c) amateur & \\
\hline & d) athlete & \\
\hline \multirow{2}{*}{ Compliance } & a) compliant & 0: compliant \\
\hline & b) not compliant & 1: not compliant \\
\hline \multirow{2}{*}{$\begin{array}{l}\text { Length of the integrated clinical and } \\
\text { rehabilitative pathway }\end{array}$} & \multirow{2}{*}{ Number of Rehabilitation days } & $0:<100$ days \\
\hline & & $1:>=100$ days \\
\hline \multirow{2}{*}{ Muscle strength test and threshold tests } & \multirow{2}{*}{ Number of tests } & $0:<2$ \\
\hline & & $1:>=2$ \\
\hline \multirow{2}{*}{ Integrated pathway Management Cost } & \multirow{2}{*}{ Value of the pathway } & $0:<3,360 €$ \\
\hline & & $1:>=3,360 €$ \\
\hline
\end{tabular}


Table 4: QCA analysis for rehabilitative pathway management cost.

\begin{tabular}{|c|c|c|c|}
\hline \multicolumn{4}{|c|}{ Modello_1_OUTCOME COST } \\
\hline & & Raw Coverage & Consistency \\
\hline 1 & $\begin{array}{c}\text { COMPLIANCE*age*SPORTINESS* LENGTH REHABILITATION * strength } \\
\text { test }\end{array}$ & 0.154 & 1 \\
\hline 2 & $\begin{array}{l}\text { COMPLIANCE*age *LENGTH REHABILITATION *SOGLIA* STRENGTH } \\
\text { TEST }\end{array}$ & 0.077 & 1 \\
\hline 3 & $\begin{array}{c}\text { COMPLIANCE* sportiness * LENGTH REHABILITATION * THRESHOLD } \\
\text { TEST* STRENGTH TEST }\end{array}$ & 0.269 & 1 \\
\hline 4 & $\begin{array}{c}\text { compliance } * \text { age } * \text { sportiness } * \text { LENGTH REHABILITATION * } \\
\text { THRESHOLD TEST } * \text { strength test }\end{array}$ & 0.038 & 1 \\
\hline 5 & $\begin{array}{l}\text { COMPLIANCE*age* SPORTINESS * length rehabilitation * threshold } \\
\text { test* STRENGTH TEST }\end{array}$ & 0.038 & 1 \\
\hline 6 & $\begin{array}{l}\text { COMPLIANCE*AGE* SPORTINESS* LENGTH REHABILITATION * } \\
\text { threshold test* STRENGTH TEST }\end{array}$ & 0.038 & 1 \\
\hline \multirow[t]{3}{*}{7} & $\begin{array}{c}\text { Compliance*AGE* SPORTINESS* LENGTH REHABILITATION * } \\
\text { THRESHOLD TEST* STRENGTH TEST }\end{array}$ & 0.038 & 1 \\
\hline & Solution Coverage 0.615 & & \\
\hline & Solution Consistency 1 & & \\
\hline
\end{tabular}

\section{Discussion and Conclusion}

In the current era, where the availability of large amounts of clinical data gathered during care delivery is rapidly increasing, as well as the ability to access, process, link, and analyze these data in efficient ways, alternative sources to supplement evidence from RCTs look promising. Among all the information that could derive from RWD, one of the most important topics to analyze consist of the management costs related to a specific integrated clinical or rehabilitative pathway. The knowledge of the economic resources' absorption could contribute to fully understand the pathways as well as the choices and behaviors involved in those pathways. Economic information provides the tools for developing effective and efficient policy strategies and addressing potential tradeoffs between the goals of increasing social welfare and improving the distribution of healthcare delivery across individuals and population groups.

Within the setting of ACL integrated rehabilitative pathway, results reported that strong compliance and a high level of sportiness could be considered among the main factors that cause an increase in the total cost of managing the rehabilitation pathway for a patient with ACL rupture, confirming the information found in literature [14-16]. In fact, orthopedic surgery often requires many months of rehabilitation to achieve a successful outcome, regardless of subspecialty, with an important impact on the overall resources' absorption [28]. For patients practicing sports by profession, the rehabilitation pathway could be more intense and costly, as it is their priority, to recover their normal motor and sports conditions as soon as possible [8]. Thanks to the QCA it is possible to integrate these results, identifying all combinations of factors that are determining the emergence of a certain results. For example, more consistent QCA analysis solutions show that compliance has an important influence on the absorption of economic resources by patients, but also higher levels of sportiness related to young age of patients, determining higher pathway costs. The results obtained and the findings of this study could give a contribute useful to enlarge the existing research, regarding the use of RWD in the healthcare sector, with particular focus on the rehabilitation programs, thus considering a larger sample and producing results that could be replicable. Although the relevance of this topic, healthcare stakeholders often do not have enough information on outcomes to take decisions, being well-informed. In this context, knowing which are the factors that have a positive or negative influence on the integrated patients' rehabilitative pathway, could be useful to optimize economic resources, as well as for the achievement of an adequate effectiveness, thus becoming more efficient and effective [6]. This, in the Italian setting, is becoming more and more important, due to the recent re-consideration of the integrated clinical and rehabilitative pathways, after the COVID-19 pandemic, for the re-organization of the healthcare delivery of services.

\section{Clinical Messages}

1. From an economic point of view, rehabilitation should rely on country-oriented and real-world data, thus providing stakeholders with valuable information about the safety and effectiveness of a clinical and rehabilitative pathway, in representative large and heterogeneous populations.

2. The knowledge of the economic resources' absorption could contribute to fully understand the integrated clinical and 
rehabilitative pathways, as well as the choices and behaviors involved in those pathways.

3. Economic information provides the tools for developing effective and efficient policy strategies and addressing potential trade-offs between the goals of increasing social welfare and improving the distribution of healthcare delivery across individuals and population groups.

4. The proposed paper may represent a significant contribution in the specific setting of rehabilitation, because literature evidence most focus the attention on the inpatients integrated clinical pathway, not considering the outpatients' pathway after discharge.

\section{Acknowledgement}

The Authors of the present paper would like to sincerely thank all the healthcare professionals involved in the data retrieval, useful for the achievement of the study objective.

\section{Competing Interests}

The Authors declare that there is no conflict of interest

\section{Funding}

This research received no specific funding from any agency, commercial or not-for-profit sectors.

\section{Availability of Data and Materials}

The datasets used and/or analysed during the current study are available from the corresponding author on reasonable request.

\section{Author Contributions}

Davide Crisci and Emanuela Foglia conceives the study; Daniele Bellavia, Federica Asperti and Lucrezia Ferrario performed the statistical analysis; Lorella Cannavacciuolo performed the QcA analyses; Lucrezia Ferrario and Lorella Cannavacciuolo wrote the draft of paper; Emanuela Foglia and Cristina Ponsiglione critically revised the paper. All authors discussed the results and commented on the manuscript.

\section{References}

1. Berguer R (2004) The evidence thing. Ann Vasc Surg 18: 265-270.

2. Celine M, Robin E, Horvath V, Catriona Manville, Jonathan Cave, et al. (2014) Health and Healthcare: Assessing the Real World Data Policy Landscape in Europe. Santa Monica, CA: RAND Corporation.

3. Garrison LP, Neumann PJ, Erickson P, Marshall D, Mullins CD (2007) Using real-world data for coverage and payment decisions: The ISPOR real-world data task force report. Value in health 10(5): 326-335.

4. Anke-Peggy Holtorf, John B Watkins, C Daniel Mullins, Diana Brixner (2008) Incorporating observational data into the formulary decisionmaking process - summary of a roundtable discussion. J Manag Care Pharm 14(3): 302-308.
5. Nguyen TH, Trocio J, Kowal S, Ferrufino CP, Munakata J, et al. (2016) Leveraging Real-World Evidence in Disease-Management DecisionMaking with a Total Cost of Care Estimator. Am Health Drug Benefits 9(9): 475-485.

6. Justo N, Espinoza M, Ratto B, Rosselli D, Ovcinnikova O, et al. (2018) Real World Evidence in healthcare decision-making: Global trends and case studies from Latin America. White paper. Stockholm, Sweden.

7. Nas K, Yazmalar L, Şah V, Aydin A, Öneș K (2015) Rehabilitation of Spinal Cord Injuries. World Journal of Orthopedics 6(1): 8.

8. De Valk E, Moen M, Winters M, Bakker E, Tamminga R, et al. (2013) Preoperative Patient and Injury Factors of Successful Rehabilitation After Anterior Cruciate Ligament Reconstruction With Single-Bundle Techniques. Arthroscopy: The Journal of Arthroscopic \& Related Surgery 29(11): 1879-1895

9. Wierike S, Van der Sluis A, Van den Akker-Scheek I, Elferink-Gemser M, Visscher C (2012) Psychosocial factors influencing the recovery of athletes with anterior cruciate ligament injury: A systematic review. Scandinavian Journal of Medicine \& Science in Sports 23(5): 527-540.

10. Christino M, Fleming B, Machan J, Shalvoy R (2016) Psychological Factors Associated With Anterior Cruciate Ligament Reconstruction Recovery. Orthopaedic Journal of Sports Medicine 4(3).

11. Czuppon S, Racette B, Klein S, Harris-Hayes M (2013) Variables associated with return to sport following anterior cruciate ligament reconstruction: a systematic review. British Journal of Sports Medicine 48 (5): 356-364.

12. Thomeé R, Kaplan Y, Kvist J, Myklebust G, Risberg MA, et al. (2011) Muscle strength and hop performance criteria prior to return to sports after ACL reconstruction, Knee Surgery, Sports Traumatology. Arthroscopy 19(11): 1798-1805.

13. Duarte A, Bojke C, Cayton Q Salawu A, Case B, et al. (2018) Impact of specialist rehabilitation services on hospital length of stay and associated costs. Eur J Health Econ 19(7): 1027-1034.

14. Scott SM, Perry MA, Gisela S (2017) Not always a straight path: patients' perspectives following anterior cruciate ligament rupture and reconstruction. Disability and Rehabilitation 40(19): 2311-2317.

15. Essery R, Geraghty AWA, Kirby S, Yardley L (2016) Predictors of adherence to home-based physical therapies: a systematic review. Disability and Rehabilitation 39(6): 519-534.

16. Brewer B (1998) Adherence to Sport Injury Rehabilitation Programs. Journal of Applied Sport Psychology 10(1): 70-82.

17. Iuga A0, McGuire MJ (2004) Adherence and health care costs. Risk Manag Healthc Policy 7: 35-44.

18. Croce D, Lazzarin A, Rizzardini G, et al. (2016) HIV Clinical Pathway: A New Approach to Combine Guidelines and Sustainability of AntiRetroviral Treatment in Italy. PlosOne.

19. Lingsma HF, Bottle A, Middleton S, Kievit K, Steyerberg EW, et al. (2018) Evaluation of hospital outcomes: the relation between length-of-stay, readmission, and mortality in a large international administrative database. BMC Health Serv Res 18: 116.

20. Risberg M, Lewek M, Snyder-Mackler L (2004) A systematic review of evidence for anterior cruciate ligament rehabilitation, how much and what type. Phys Ther Sport 5: 125-145.

21. Vandenbroucke JP, Von Elm E, Altman DG, Peter C Gøtzsche, Cynthia D Mulrow, et al. (2007) Strengthening the Reporting of Observational Studies in Epidemiology (STROBE): Explanation and Elaboration. Ann Intern Med 147: W-163-W-194. 
22. Cohen J (1988) Statistical power analysis for the behavioral sciences $\left(2^{\text {nd }}\right.$ Edn.)., Hillsdale, NJ: Erlbaum.

23. Ragin CC (1987) The comparative method: Moving beyond qualitative and quantitative methods. Berkeley: University of California.

24. Ragin CC (2000) Fuzzy-set social science, University of Chicago Press.

25. Marx A, Cambré B, Rihoux B (2013) Chapter 2 crisp-set qualitative comparative analysis in organizational studies. In Configurational theory and methods in organizational research, Emerald Group Publishing Limited, p. 23-47.

ISSN: 2574-1241

DOI: 10.26717/BJSTR.2022.41.006564

Lucrezia Ferrario. Biomed J Sci \& Tech Res

(C) (P) This work is licensed under Creative

Submission Link: https://biomedres.us/submit-manuscript.php
26. Vis B (2012) The Comparative Advantages of fsQCA and Regression Analysis for Moderately Large-N Analyses. Sociological Methods and Research 41(1): 168-198.

27. Poveda AC, Martínez CIP (2013) Qualitative comparative analysis (QCA): an application for the industry. Quality \& Quantity 47(3): 1315-1321.

28. Flanigan DC, Everhart JS, Glassman AH (2015) Psychological Factors Affecting Rehabilitation and Outcomes Following Elective Orthopaedic Surgery. J Am Acad Orthop Surg 23(9): 563-570. 